Artículo

\title{
La densidad de siembra en el crecimiento de la verdolaga
}

\author{
Erika Lagunes-Fortiz ${ }^{1 \S}$ \\ Clemente Villanueva-Verduzco ${ }^{1}$ \\ Edgar Ricardo Lagunes-Fortiz ${ }^{1}$ \\ Erika Janet Zamora-Macorra ${ }^{1}$ \\ Norma Ávila-Alistac ${ }^{1}$ \\ Evert Villanueva-Sánchez ${ }^{2}$
}

${ }^{1}$ Universidad Autónoma Chapingo. Carretera México-Texcoco km 38.5, Chapingo, Texcoco, Estado de México. CP. 56230. Tel. 595 9521500, ext. 6133. ${ }^{2}$ CONACYT-Universidad Autónoma Chapingo. Laboratorio Nacional de Investigación y Servicio Agroalimentario y Forestal. Carretera México-Texcoco km 38.5, Chapingo, Estado de México, México. CP. 56230.

${ }^{\S}$ Autora para correspondencia: elagunesf@chapingo.mx.

\section{Resumen}

En México existen más de 358 especies de plantas, de las que únicamente se consumen hojas y tallos tiernos, conocidas como 'quelites'. Dado el potencial productivo, económico y nutrimental de estas especies, entre ellas la verdolaga, es preciso determinar factores que afecten su crecimiento y desarrollo. La verdolaga constituye una alternativa como hortaliza debido a su potencial nutrimental, productivo y económico; su rendimiento estará determinado principalmente por el desarrollo de tallos y hojas, por lo que el conocimiento generado sobre los factores que afectan su crecimiento resulta de gran importancia agronómica. En la presente investigación se evaluó el efecto de tres densidades de siembra en el crecimiento de la verdolaga. Se consideraron los datos correspondientes a tres etapas fenológicas del desarrollo de la verdolaga (desarrollo vegetativo, madurez vegetativa y floración) para determinar si existían diferencias significativas entre las medias de los tratamientos evaluados mediante un Anova y para determinar si existía homogeneidad de varianza se llevó a cabo el método Levene. Las plantas sembradas a una densidad de siembra alta (295 plantas $\mathrm{m}^{-1}$ lineal) tuvieron mayor altura, menor diámetro de tallo, menor número de hojas (grandes y chicas) y menor desarrollo de las ramas (longitud de ramas) en comparación con aquellas sembradas en densidades de siembra media y baja ( 250 y 134 plantas $\mathrm{m}^{-1}$ lineal); sin embargo, el número de hojas en las ramas depende del nivel del dosel donde éstas se localicen. La densidad de siembra es un factor que contribuye a determinar el crecimiento de la verdolaga "Chapingo".

Palabras clave: etapas fenológicas, quelites, verdolaga.

Recibido: enero de 2021

Aceptado: marzo de 2021 


\section{Introducción}

En México existen más de 500 especies de plantas comestibles silvestres, cuyo uso se remonta a épocas prehispánicas dada su gran relevancia cultural, culinaria y nutrimental, incluyendo los quelites, del náhuatl 'quiltil', de los cuales únicamente se consumen las hojas, tallos y flores tiernas (Bye y Linares, 2002; Balcázar-Quiñones et al., 2020), pues son una importante fuente de nutrimentos $(\mathrm{Ca}, \mathrm{K}, \mathrm{Mg}, \mathrm{Fe}, \mathrm{Mn}, \mathrm{Zn}, \mathrm{P})$, proteína, fibra dietética, vitaminas A, C y compuestos bioactivos, tales como fenoles, betalaínas, flavonoides, alcaloides, omega 3, omega 6, carotenos, ácido ascórbico, $\alpha$-tocoferol, glutatión, etc., los cuales le confieren a estas plantas una capacidad antioxidante elevada, además de aportar a los platillos diferentes aromas, colores y sabores (Bye y Linares, 2002; Santiago-Saenz et al., 2019; Balcázar-Quiñones et al., 2020).

Dado su elevado potencial productivo y a que existe una ancestral cultura de consumo de algunos de estos cultivos (verdolagas, quintoniles, pápalos, epazotes, etc.) en la población mexicana del centro del país, en donde existen 25 millones de habitantes tan solo en la Ciudad y el Estado de México (INEGI, 2015), las Instituciones de Enseñanza e Investigación deben desarrollar tecnología, variedades, sistemas intensivos de producción y prácticas agronómicas que proporcionen un conocimiento integral de las diferentes especies de quelites y que contribuyan a impulsar el óptimo aprovechamiento de su potencial económico y como planta alimenticia.

En México, la producción de verdolaga comercial se concentra en los estados de Morelos (171 ha), Ciudad de México (158 ha), Estado de México (45 ha) y Baja California (46 ha) con rendimientos que oscilan entre 12 y $14.86 \mathrm{udm} \mathrm{ha}^{-1}$ y una producción de $5474.15 \mathrm{t}$, con un valor de la producción de poco más de 26361.30 miles de pesos (SIAP, 2019).

En nuestro país, existen desde hace muchos años dos regiones productoras bien definidas (contrastantes en clima) con variedades locales criollas domesticadas bajo cultivo: la región de Xochimilco-San Gregorio-Mixquic (clima templado) y la de Cuautla, Morelos (clima cálido) (Villanueva y Ramírez, 2003). La activa y continua domesticación de la verdolaga en las diferentes regiones productoras ha permitido la obtención de genotipos con hojas más gruesas, hábitos de crecimiento erecto, mayor precocidad y resistencia a enfermedades, como lo es el cultivar 'Chapingo' de verdolaga, cuyos análisis bromatológicos han mostrado que también contiene elevados niveles de hierro, magnesio, omega 3 y omega 6 (Solís et al., 2016).

Estudios conducidos bajo invernadero (sin calefacción) durante el ciclo de invierno indican que es posible producir hasta $20 \mathrm{~kg} \mathrm{~m}^{-2}$ de hortaliza en 50 días $\left(200 \mathrm{t} \mathrm{ha}^{-1}\right)$, con una densidad de población de 500 plantas $\mathrm{m}^{-2}$ (Villanueva y Ramírez, 2003). El valor de la cosecha de verdolaga de invierno puede alcanzar hasta $\$ 8.00$ por kilogramo al productor, aunque baja drásticamente en épocas del año de su producción natural (Villanueva y Ramírez, 2003; Mera et al., 2010).

El estudio del crecimiento de la verdolaga y de los factores que lo afectan resulta de gran importancia, pues su rendimiento estará determinado en su mayoría por el desarrollo de la parte vegetativa de la planta, la cual a su vez dependerá de múltiples factores, algunos de ellos propios del genotipo, otros del ambiente y de diversas condiciones de manejo del cultivo. 
Recientemente Montoya-García et al. (2017) evaluaron el efecto de la a fertilización con N, P y K en la producción de verdolaga, encontraron que la altura y rendimiento de la verdolaga incrementan en respuesta a la aplicación de nitrógeno aplicado y a la fecha de cosecha. Montoya-García et al. (2018) reportan que es posible maximizar el contenido nutracéutico, así como la concentración de ácidos grasos y antioxidantes con fertilizaciones con nitrógeno, fósforo y potasio, por lo que factores como la fertilización no sólo influyen en el crecimiento de la planta, sino también en la potencialización de sus propiedades en beneficio de la salud.

El conocimiento sobre el crecimiento del cultivo constituye la base para la óptima planeación y ejecución de labores culturales a realizar durante el proceso productivo, a fin de obtener los mejores rendimientos. El genotipo, fecha de siembra, densidad de siembra, localidad y clima en que se desarrollen las plantas son factores que pueden influenciar la cantidad de tejido foliar, hábito de crecimiento, parte de la planta y desarrollo de otras estructuras.

Los factores más importantes que afectan la duración de las etapas de desarrollo de la verdolaga incluyen el genotipo (dentro y entre poblaciones), el ambiente (clima y suelo) y sus interacciones, debido a los cuales puede variar el hábito de crecimiento y la precocidad de la planta. Algunas otras características de la planta como el hábito de crecimiento, sabor, filotaxia y tallo, pueden variar dependiendo de si la planta se desarrolla como maleza o cultivada (Villanueva y Ramírez, 2003). El crecimiento vegetal, implica a nivel fisiológico una serie de cambios y reacciones irreversibles en la planta, de las cuales dependerán las características agronómicas finales (fenotipo) y el rendimiento potencial de los diferentes genotipos.

La evaluación del crecimiento de una planta se puede realizar a través de medidas directas (altura de la planta, el diámetro del tallo, el número de hojas, el número de ramas, área foliar, masa fresca o seca, etc.) e indirectas (tasa de asimilación neta, tasa de crecimiento del cultivo, tasa relativa de crecimiento, etc.) (Rodríguez, 2000).

La densidad de siembra es un factor que puede influir en el rendimiento de cultivos como la verdolaga, pues interfiere con eventos fisiológicos de la planta y directamente con acumulación de materia seca de los diferentes órganos de la planta, además determina la competencia entre las plantas por luz, agua y nutrimentos (Rodríguez, 2000; Ayala et al., 2004). Con base en lo anterior, el objetivo de la presente investigación fue evaluar el efecto de tres densidades de siembra sobre el crecimiento del cultivar 'Chapingo' de verdolaga, así como caracterizar su dinámica y hábito de crecimiento.

\section{Materiales y métodos}

El experimento se estableció en el Campo Agrícola Experimental de la Universidad Autónoma Chapingo, México a $19^{\circ} 29^{\prime}$ '27' latitud norte y 98 52' $23^{\prime}$ ' longitud oeste, a una altitud de 240 m. Las modificaciones hechas por García (1988) al sistema de clasificación climática de Köppen describe el sitio del establecimiento del experimento como un $\mathrm{C}(\mathrm{W})(\mathrm{w}) \mathrm{b}(\mathrm{i}$ ')g, lo cual significa que es el más seco de los templados subhúmedos con lluvias en verano, una temperatura media anual de $15.6^{\circ} \mathrm{C}$ y de $-3^{\circ}$ a $18^{\circ} \mathrm{C}$ en el mes más frío (enero), un verano fresco, la temperatura del mes más cálido es menor de $22^{\circ} \mathrm{C}$ (mayo), con poca oscilación térmica anual que varía entre $5^{\circ}$ y $7^{\circ} \mathrm{C}$. El suelo es clasificado como Mollic ustifluvent, con una profundidad de $150 \mathrm{~cm}$ y una textura franco arcillo limosa en el horizonte Ap (Soil Survey Staff, 2010). 
Se sembraron, a chorrillo, 40 surcos \pm 1 de $30 \mathrm{~m}$ de largo y una distancia entre surcos $0.6 \mathrm{~m}$ con verdolaga del cultivar 'Chapingo'. Los sitios de muestreo se establecieron al azar, de tal forma que cada unidad experimental tuviera una densidad de siembra diferente, se delimitó $1 \mathrm{~m}$ para cada unidad experimental y se contó el número de plantas en cada unidad experimental, quedando tres densidades de siembra diferentes (tratamientos): alta (295 plantas $\mathrm{m}^{-1}$ lineal), media (250 plantas $\mathrm{m}^{-1}$ lineal) y baja (134 plantas $\mathrm{m}^{-1}$ lineal). En cada unidad experimental, se tomaron los datos a 20 plantas (repeticiones).

Las variables que se describen a continuación se evaluaron cada 4 días a partir de la siembra y hasta los 48 días después de la siembra (dds). Las variables estudiadas fueron la altura total de la planta (AT), se midió en centímetros desde el nivel del suelo hasta el final del tallo principal; número de ramas (NR), se contaron las ramas que se insertaban directamente en el tallo principal; diámetro del tallo (DT), se midió en milímetros (mm) con ayuda de un Vernier, en el segundo entrenudo de la planta, justo debajo del tercer nudo; número de hojas grandes y de hojas chicas (NHG y NHCH, respectivamente), se contaron las hojas grandes y chicas de la planta, considerando como hojas grandes aquellas que medían entre 1.5 a $2 \mathrm{~cm}$ de ancho y 2.5 a $3 \mathrm{~cm}$ de largo; longitud promedio de las ramas del n nudo (LPRn), se tomó el promedio, en centímetros, de la longitud de las dos ramas que se insertaban en el n nudo del tallo principal, numerando los nudos de la base a la punta de la planta y número de hojas promedio de las ramas del n nudo (NHPn), se refiere al promedio del número total de hojas de cada una de las ramas que se insertaban en el n nudo del tallo principal, numerando los nudos de la base a la punta de la planta.

Para realizar el análisis estadístico se tomaron los datos correspondientes a tres etapas fenológicas del desarrollo de la verdolaga: desarrollo vegetativo (19 dds; 52.1 Unidades Calor, UC), madurez vegetativa (31 dds, 100.65 UC) y floración (39 dds, 39 142.55 UC) de verdolaga del cultivar 'Chapingo'. Los datos se sometieron a un análisis con el paquete SAS System for Windows 9.1 (SAS Institute, 2002), para determinar si existían diferencias estadísticamente significativas entre las medias de las tres densidades de siembra evaluadas se realizó un Anova y para determinar si existía igualdad de sus varianzas se utilizó el método Levene, ambas con un nivel de significancia de $p \leq 0.05$.

\section{Resultados y discusión}

En la Figura 1 se observa el crecimiento de la verdolaga en altura de la planta (AT), número de ramas (NR) y diámetro del tallo (DT); en la Figura 2, el número de hojas grandes (NHG) y número de hojas chicas $(\mathrm{NHCH})$, mientras que en la Figura 3, el crecimiento de las ramas del tallo principal a la altura del $1^{\mathrm{er}}$ y $4^{\text {to }}$ nudo, contando desde la base a la punta de la planta. La dinámica de crecimiento representada en dichas figuras muestra que es entre los 20 y 40 dds donde el crecimiento de la verdolaga es acelerado por lo que, aunque las variables se evaluaron cada 4 días, el Anova y la prueba de Levene de comparación de varianza se realizó con los datos obtenidos en las etapas fenológicas que abarcan dicho periodo (desarrollo vegetativo, madurez vegetativa y floración). 


\section{Altura de planta}

Durante todo el ciclo de vida de la planta, la altura incrementa constante y exponencialmente, particularmente entre los 20 y $40 \mathrm{dds}$, lo cual coindice con el inicio de la etapa de desarrollo vegetativo y hasta el inicio de la floración (Figura 1a). La prueba de comparación de medias de AT muestra que sólo durante la etapa de madurez vegetativa hubo diferencias estadísticamente significativas entre las medias de las tres densidades de siembra ( $p<0.05$, Cuadro 1). La mayor AT se obtuvo en la densidad de siembra alta (295 plantas $\mathrm{m}^{-1}$ lineal) respecto a las densidades media y baja (250 y 134 plantas $\mathrm{m}^{-1}$ lineal, respectivamente).

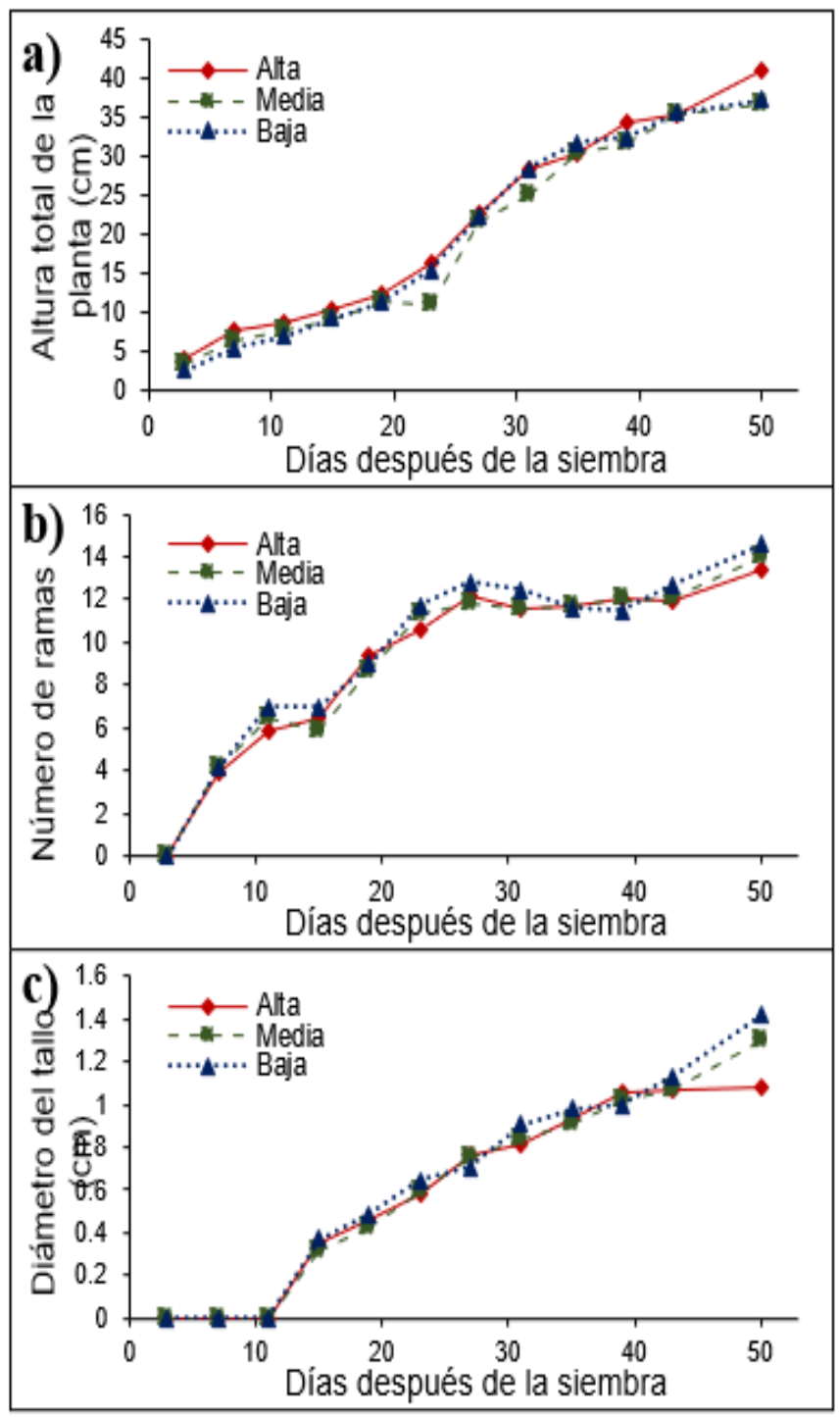

Figura 1. Dinámica del crecimiento de a) altura total de la planta $(\mathrm{cm})$, b) número de ramas y c) diámetro del tallo $(\mathrm{en} \mathrm{cm})$ de la verdolaga en tres densidades de siembra (alta: 295; media: 250 y baja: 134 plantas $\mathrm{m}^{-1}$ lineal, respectivamente). 
Cuadro 1. Anova de altura total de la planta (AT, en $\mathrm{cm})$, número de ramas (NR), diámetro del tallo (DT, en cm), número de hojas grandes (NHG) y número de hojas chicas (NHCH) a los 19 dds (desarrollo vegetativo), a los 31 dds (madurez vegetativa) y a los 39 dds (inicio de la floración) de verdolaga de la variedad 'Chapingo' sembrada en tres densidades de siembra (alta: 295; media: 250 y baja: 134 plantas $\mathrm{m}^{-1}$ lineal, respectivamente).

\begin{tabular}{|c|c|c|c|c|c|c|c|c|c|c|}
\hline \multirow{2}{*}{$\begin{array}{c}\text { Densidad } \\
\text { de } \\
\text { siembra }\end{array}$} & \multicolumn{2}{|r|}{ AT } & \multicolumn{2}{|r|}{ NR } & \multicolumn{2}{|r|}{ DT } & \multicolumn{2}{|r|}{ NHG } & \multicolumn{2}{|c|}{$\mathrm{NHCH}$} \\
\hline & $\mathrm{M}$ & $\begin{array}{c}\text { Anova } \\
\text { (valor F/Pr) }\end{array}$ & M & $\begin{array}{c}\text { Anova } \\
\text { (valor F/Pr) }\end{array}$ & $\mathrm{M}$ & $\begin{array}{c}\text { Anova } \\
\text { (valor F/Pr) }\end{array}$ & M & $\begin{array}{c}\text { Anova } \\
\text { (valor F/Pr) }\end{array}$ & $\mathrm{M}$ & $\begin{array}{c}\text { Anova } \\
\text { (valor F/Pr) }\end{array}$ \\
\hline \multicolumn{11}{|c|}{ Desarrollo vegetativo } \\
\hline Alta & 12.29 & $0.2 / 0.8197$ & 9.4 & $0.45 / 0.6419$ & 0.45 & $0.64 / 0.5335$ & 10.75 & $2.02 / 0.1416$ & 41 & $4.39 / 0.0169$ \\
\hline Media & 11.6 & & 8.65 & & 0.42 & & 10.55 & & 38.4 & \\
\hline Baja & 11.4 & & 9 & & 0.48 & & 12.25 & & 54.3 & \\
\hline \multicolumn{11}{|c|}{ Madurez vegetativa } \\
\hline Alta & 28.35 & $3.68 / 0.0313$ & 11.55 & $2.68 / 0.077$ & 0.81 & $2.62 / 0.0813$ & 11.5 & $1.31 / 0.2768$ & 107.15 & $0.45 / 0.6376$ \\
\hline Media & 25.26 & & 11.6 & & 0.84 & & 12.15 & & 111.45 & \\
\hline Baja & 28.6 & & 12.45 & & 0.9 & & 13.75 & & 114.3 & \\
\hline \multicolumn{11}{|c|}{ Floración } \\
\hline Alta & 34.51 & $2.54 / 0.0877$ & 12.05 & $0.66 / 0.5205$ & 1 & $0.64 / 0.5335$ & 10.15 & $2.79 / 0.07$ & 227.1 & $0.04 / 0.9633$ \\
\hline Media & 31.69 & & 12 & & 1.02 & & 12.35 & & 224.8 & \\
\hline Baja & 32.48 & & 11.5 & & 1.05 & & 11.5 & & 222.5 & \\
\hline
\end{tabular}

$\mathrm{M}=$ media. Grupo de datos en negritas indican diferencias estadísticamente significativas entre ellos $(p<0.05)$.

Un análisis preliminar de los datos reveló que la dispersión de las observaciones en las tres densidades de siembra podría ser distinta, por lo que se realizó una prueba de Levene. Se concluyó que las varianzas son estadísticamente distintas por lo que la densidad de siembra es un factor que contribuye a determinar la altura de planta de la verdolaga (Cuadro 2).

En este sentido, Rodríguez (2000) reporta que el incremento en la densidad de población genera la producción de plantas más largas, mientras que Favaro y Pilatti (1997) indican que plantas de tomate sembradas en densidad de siembras altas, originan en sus estratos inferiores una mayor proporción de luz rojo lejano $(730 \mathrm{~nm})$, afectando la actividad del fitocromo, que se expresa por un incremento en la longitud de los entrenudos y por consiguiente en la altura de la planta.

\section{Número de ramas}

Noda y Gildardo (2008) al estudiar el crecimiento de Morus alba en diferentes densidades de siembra, reportan que, las plantas desarrollan mayor número de ramas en bajas densidades de siembra, pues la poca competencia por luz y la disposición de radiación solar directa favorece el desarrollo de las ramas laterales; sin embargo, en la presente investigación, no se encontraron diferencias estadísticamente significativas en el número de ramas (NR, Cuadro 1) determinadas por la densidad de siembra, debido probablemente a que la verdolaga 'Chapingo' al ser un material previamente seleccionado, cuenta con características estructurales bien definidas, como hábito de crecimiento erecto, filotaxia opuesta decusada, tallo largo, grueso, suculento de color verde y disposición de sus ramas laterales opuestas, la cual conserva de forma muy estable. 
Entre los 20 y 30 dds el desarrollo de ramas es acelerado (periodo que coindice con la etapa de desarrollo vegetativo) y posteriormente incrementa, pero en menor magnitud, permaneciendo estable a partir de los 30 dds y hasta el final de su ciclo de vida. La verdolaga 'Chapingo' llega a desarrollar de 8 a 9 ramas en la etapa de desarrollo vegetativo, 12 ramas en el inicio de la floración (Cuadro 2) y hasta 14 ramas al final de su ciclo (48 dds, Figura 1).

Cuadro 2. Desviación estándar de la altura total de la planta (AT, en cm), número de ramas (NR), diámetro del tallo (DT, en cm), número de hojas grandes (NHG) y número de hojas chicas (NHCH) a los 19 dds (desarrollo vegetativo), a los 31 dds (madurez vegetativa) y a los 39 dds (floración) de verdolaga de la variedad 'Chapingo' sembrada en tres densidades de siembra (alta: 295; media: 250 y baja: 134 plantas $\mathrm{m}^{-1}$ lineal, respectivamente).

\begin{tabular}{|c|c|c|c|c|c|c|c|c|c|c|}
\hline \multirow{2}{*}{$\begin{array}{c}\text { Densidad } \\
\text { de } \\
\text { siembra }\end{array}$} & \multicolumn{2}{|r|}{ AT } & \multicolumn{2}{|r|}{ NR } & \multicolumn{2}{|r|}{ DT } & \multicolumn{2}{|r|}{ NHG } & \multicolumn{2}{|r|}{$\mathrm{NHCH}$} \\
\hline & DS & $\begin{array}{c}\text { Levene } \\
\text { (valor F/Pr) }\end{array}$ & DS & $\begin{array}{c}\text { Levene } \\
\text { (valor F/Pr) }\end{array}$ & DS & $\begin{array}{c}\text { Levene } \\
\text { (valor F/Pr) }\end{array}$ & DS & $\begin{array}{c}\text { Levene } \\
\text { (valor F/Pr) }\end{array}$ & DS & $\begin{array}{c}\text { Levene } \\
\text { (valor F/Pr) }\end{array}$ \\
\hline \multicolumn{11}{|c|}{ Desarrollo vegetativo } \\
\hline Alta & 4.94 & $0.06 / 0.9373$ & 2.6 & $0.05 / 0.9536$ & 0.2 & $0.09 / 0.9142$ & 2.27 & $1.64 / 0.2024$ & 16.62 & $0.37 / 0.693$ \\
\hline Media & 4.77 & & 2.46 & & 0.17 & & 2.61 & & 18.64 & \\
\hline Baja & 4.42 & & 2.47 & & 0.18 & & 3.7 & & 19.27 & \\
\hline \multicolumn{11}{|c|}{ Madurez vegetativa } \\
\hline Alta & 4.28 & $2.94 / 0.061$ & 1.5 & $0.53 / 0.5896$ & 0.19 & $0.77 / 0.465$ & 2.37 & $1.8 / 0.1741$ & 25.4 & $0.27 / 0.768$ \\
\hline Media & 3.17 & & 1.43 & & 0.1 & & 6.96 & & 25.26 & \\
\hline Baja & 5.28 & & 1.19 & & 0.1 & & 2.68 & & 20.75 & \\
\hline \multicolumn{11}{|c|}{ Floración } \\
\hline Alta & 5.35 & $3.48 / 0.0377$ & 1.68 & $0.46 / 0.6364$ & 0.2 & $0.09 / 0.9142$ & 2.77 & $1.67 / 0.1978$ & 34.13 & $0.99 / 0.3786$ \\
\hline Media & 3.49 & & 1.84 & & 0.17 & & 2.91 & & 62.67 & \\
\hline Baja & 3.79 & & 1.47 & & 0.18 & & 3.65 & & 60.59 & \\
\hline
\end{tabular}

$\mathrm{M}=$ media. Grupo de datos en negritas indican grupo de varianzas con diferencias estadísticamente significativas entre ellos $(p<0.05)$.

Autores mencionan que uno de los factores más sensibles a las variaciones de densidad es el número de ramas por planta, un incremento en la población de plantas produce una disminución en el número de ramas (Ayala et al., 2004; Kakiuchi y Kobata, 2004) lo cual coincide con los resultados obtenidos en la presente investigación pues el número de ramas fue menor en las plantas de verdolaga sembradas en la densidad de siembra alta en comparación con las densidades de siembra media y baja.

\section{Diámetro del tallo}

A partir de los 10 dds el diámetro del tallo incrementa exponencialmente, de los 20 y 40 dds se presenta un crecimiento acelerado similar en las tres densidades de siembra, el crecimiento se detiene a partir de los 43 dds (etapa de desarrollo de cápsulas) en la densidad de siembra alta (295 plantas $\mathrm{m}^{-1}$ lineal) (Figura 1c). No se encontraron diferencias altamente significativas en el diámetro del tallo entre las verdolagas sembradas en las tres densidades de siembra (Cuadro 1). 
Según García y Watson (2003), el grosor del tallo depende del genotipo, las condiciones ambientales y nutricionales del suelo, afirma que en maíz la resistencia que presenta la planta al acame, depende en gran medida del diámetro del tallo y que además el diámetro del tallo tiende a disminuir cuando se aumenta la densidad de siembra, debido a la competencia entre las plantas.

Las altas densidades de siembra y la competencia por luz con las malezas provocan una elongación de los tallos, entrenudos más largos y plantas más altas, reduciendo el grosor de los tallos y aumentando las posibilidades de acame de las plantas, esto coincide al observar los resultados obtenidos en la presente investigación a los 48 dds de la verdolaga sembradas en tres densidades de siembra distintas. El diámetro del tallo es un parámetro de gran importancia ya que además de contener los conductos que canalizan el transporte de agua y nutrientes a los diferentes órganos de la planta, también influirá en la resistencia al doblamiento de la planta cuando son afectados por factores externos como el viento o la lluvia. El cultivar 'Chapingo' de verdolaga, tiene un crecimiento erecto, por lo que un óptimo engrosamiento del tallo favorecerá el desarrollo de todos los órganos estructurales de la planta.

\section{Número de hojas}

En las tres densidades de siembra evaluadas, a partir de los 10 dds y hasta el final del ciclo de vida de la verdolaga, el número de hojas grandes (NHG) se mantiene entre 10 a 18 hojas grandes, siendo a los 27 dds donde existe la mayor cantidad de hojas grandes (18 hojas) lo cual coincide con el término de la etapa de desarrollo vegetativo, posteriormente algunas hojas caen (principalmente aquellas localizadas en la base de las ramas inferiores), evento que coincide con la etapa de insinuación (desarrollo de botones florales visibles en la parte terminal del tallo principal) y la floración, finalmente hacia el final del ciclo de vida de la planta, el número de hojas grandes se mantiene entre las 10 y 14 hojas según la densidad de siembra (Figura 2a).

La dinámica del desarrollo de las hojas chicas $(\mathrm{NCH})$ es diferente (Figura $2 \mathrm{~b}$ ) pues a partir del inicio de la etapa de desarrollo vegetativo (18 dds) y hasta la etapa de insinuación (35 dds) el número de hojas chicas es constante en las tres densidades de siembra; sin embargo de 35 a 48 dds (etapa de maduración de la semilla) el número de hojas chicas incrementa, debido quizás a la demanda que representa la producción de fotoasimilados para el desarrollo de estructuras reproductivas de la planta durante las etapas de insinuación y maduración de la semilla.
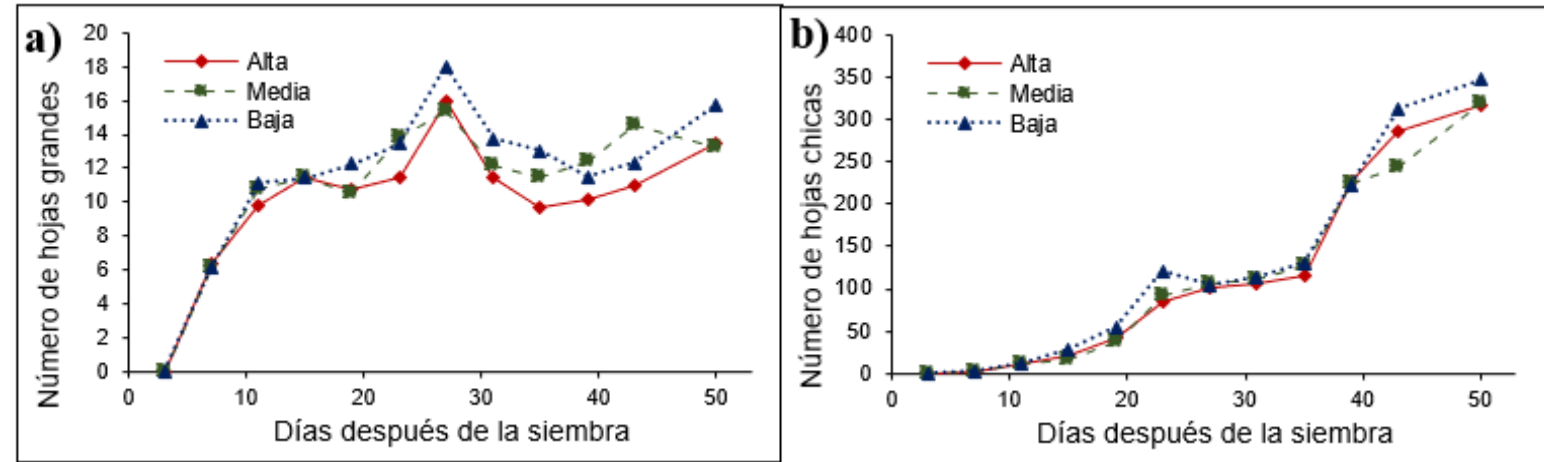

Figura 2. Dinámica de crecimiento de a) número de hojas grandes y c) número de hojas chicas de la verdolaga en tres densidades de siembra (alta: 295; media: 250 y baja: 134 plantas $\mathrm{m}^{-1}$ lineal, respectivamente). 
Las plantas de verdolaga sembradas en la densidad de siembra baja (134 plantas $\mathrm{m}^{-1}$ lineal) son las que alcanzan la mayor cantidad de hojas grandes y chicas, seguidas de aquellas sembradas en densidades de siembra media y alta, comportamiento que coindice con lo reportado por Díaz et al. (1999), quienes señalan que la producción de biomasa disminuye a medida que la densidad aumenta, debido a la mayor competencia entre las plantas por luz, $\mathrm{CO}_{2}$, agua y minerales.

\section{Longitud y número de hojas de las ramas}

Durante el desarrollo de la verdolaga se desarrollaron un total de 8 nudos en el tallo principal, sobre los cuales se desarrollaron dos ramas laterales, así como hojas nuevas en dichas ramas. En el presente estudio sólo se midieron aquellas ramas localizadas en el $1^{\mathrm{er}}$ y $4^{\text {to }}$ nudo (numerando de la base a la punta de la planta) debido a que son las que se desarrollan en mayor magnitud respecto a las ramas de los nudos superiores.

El crecimiento de las ramas y hojas en el $1^{\mathrm{er}}$ nudo ( $\mathrm{LPR}_{1}$ y $\mathrm{NHP}_{1}$, respectivamente) comienza a ser notable a partir de los 10 dds (Figura 3a y 3b). Por otro lado, el crecimiento de las ramas y hojas del $4^{\text {to }}$ nudo ( $\mathrm{LPR}_{4}$ y NHP 4 , respectivamente) se presenta entre los 15 y 20 dds (Figura 3c y d); es decir, durante la etapa de desarrollo vegetativo.

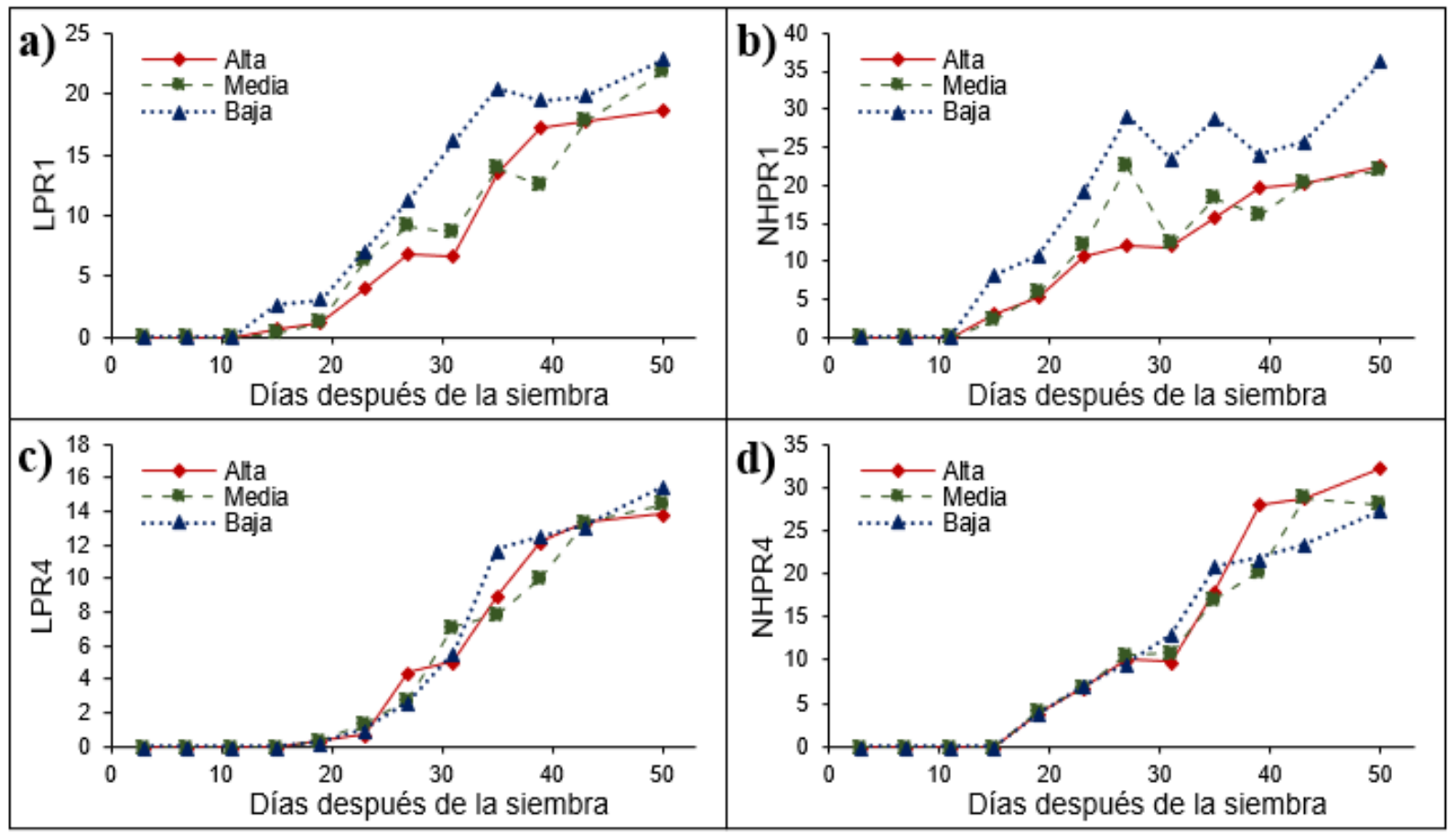

Figura 3. Dinámica de crecimiento de la longitud promedio de las dos ramas del nudo a) 1 (LPR 1 y c) $4\left(\mathrm{LPR}_{4}\right)$ y número de hojas promedio de las dos ramas del nudo a) $1\left(\mathrm{NHP}_{1}\right)$ y d) 4 (NHP $)$ de la verdolaga en tres densidades de siembra (alta: 295; media: 250 y baja: 134 plantas m m $^{-1}$ lineal, respectivamente).

En la variable $\mathrm{LPR}_{1}$, se encontraron diferencias estadísticamente significativas entre las tres densidades de siembra ( $p<0.05$ ), en las fases de desarrollo vegetativo, madurez vegetativa $\mathrm{y}$ floración, mientras que para la variable $\mathrm{NHP}_{1}$ se encontraron diferencias estadísticamente 
significativas $(p<0.05)$ entre las densidades de siembra evaluadas en las etapas de desarrollo vegetativo, madurez vegetativa, pero no así en la etapa de floración. Para el caso de las variables $\mathrm{LPR}_{4}$ y $\mathrm{NHP}_{4}$ no se encontraron diferencias estadísticamente significativas entre las medias de las tres densidades de siembra evaluadas con excepción de la variable LPR $_{4}$ en 1 etapa de madurez vegetativa ( $p<0.05$, Cuadro 3).

Cuadro 3. Longitud y número de hojas de las ramas a los 19 dds (desarrollo vegetativo), a los 31 dds (madurez vegetativa) y a los 39 dds (floración) de verdolaga de la variedad 'Chapingo' sembrada en tres densidades de siembra (alta: 295; media: 250 y baja: 134 plantas $\mathrm{m}^{-1}$ lineal, respectivamente).

\begin{tabular}{|c|c|c|c|c|c|c|c|c|}
\hline \multirow{2}{*}{$\begin{array}{l}\text { Densidad de } \\
\text { siembra }\end{array}$} & \multicolumn{2}{|r|}{$\mathrm{LPR}_{1}$} & \multicolumn{2}{|r|}{$\mathrm{NHP}_{1}$} & \multicolumn{2}{|r|}{$\mathrm{LPR}_{4}$} & \multicolumn{2}{|r|}{$\mathrm{NHP}_{4}$} \\
\hline & M & $\begin{array}{c}\text { Anova (valor } \\
\text { F/Pr) }\end{array}$ & M & $\begin{array}{c}\text { Anova (valor } \\
\text { F/Pr) }\end{array}$ & M & $\begin{array}{c}\text { Anova } \\
\text { (valor F/Pr) }\end{array}$ & M & $\begin{array}{c}\text { Anova (valor } \\
\text { F/Pr) }\end{array}$ \\
\hline \multicolumn{9}{|c|}{ Desarrollo vegetativo } \\
\hline Alta & 1.13 & $8.17 / 0.0008$ & 5.35 & $14.187 /<0.0001$ & 0.3 & $0.44 / 0.6488$ & 3.85 & $0.1 / 0.9083$ \\
\hline Media & 1.28 & & 5.9 & & 0.33 & & 4.1 & \\
\hline Baja & 3.1 & & 10.8 & & 0.26 & & 3.8 & \\
\hline \multicolumn{9}{|c|}{ Madurez vegetativa } \\
\hline Alta & 6.6 & $17.34 /<0.0001$ & 12.1 & $7.86 / 0.001$ & 5.06 & $3.29 / 0.0444$ & 9.9 & $3.06 / 0.0546$ \\
\hline Media & 8.54 & & 12.35 & & 7.15 & & 10.75 & \\
\hline Baja & 16.1 & & 23.35 & & 5.63 & & 13 & \\
\hline \multicolumn{9}{|c|}{ Floración } \\
\hline Alta & 17.22 & 4.4/0.0169 & 19.75 & 2.49/0.0916 & 12.23 & $3.04 / 0.0558$ & 28 & $2.59 / 0.0836$ \\
\hline Media & 12.49 & & 16.1 & & 10.1 & & 20.4 & \\
\hline Baja & 19.46 & & 24 & & 12.6 & & 21.8 & \\
\hline
\end{tabular}

LPR $n=$ longitud promedio de las dos ramas del $n$ nudo; NHP $n=$ número de hojas promedio de las dos ramas del $n$ nudo. Grupo de datos en negritas indican diferencias estadísticamente significativas entre ellos $(p<0.05)$.

Según la prueba de comparación de varianzas para las variables $\mathrm{LPR}_{1}, \mathrm{NHPR}_{1}, \mathrm{LPR}_{4}$ y $\mathrm{NHPR}_{4}$, sólo en pocos casos las hubo diferencias estadísticamente significativas, por lo que la densidad de siembra influye en el número de ramas y hojas que se crecen en la planta (Cuadro 4). Poulain (1984) señala que altas densidades de siembra generan plantas más altas, pero con menor número de ramificaciones, tal como ocurrió en el crecimiento de las ramas en el $1^{\mathrm{er}} \mathrm{y} 4^{\text {to }}$ nudo $\left(\mathrm{LPR}_{1} \mathrm{y}\right.$ $\mathrm{LPR}_{4}$, respectivamente) donde las plantas sembradas en la densidad de siembra alta alcanzaron menor longitud en comparación con aquellas sembradas en las densidades media y baja.

Las ramas del primer nudo de aquellas plantas sembradas en baja densidad contenían el mayor número de hojas $\left(\mathrm{NHP}_{1}\right)$ en comparación con aquellas de las densidades media y baja, debido posiblemente a que su longitud y disponibilidad de espacio les permitió producir mayor cantidad de hojas. Por otro lado, el número de hojas de las ramas del $4^{\text {to }}$ nudo (NHPR 4 ) fue mayor en las plantas de verdolaga sembradas en la densidad de siembra alta, en comparación con las densidades media y baja, debido probablemente a que el nivel del dosel donde se localizaban influyó en el desarrollo de las hojas para abastecer la demanda de fotoasimilados necesarios para la formación de estructuras propias de las etapas fenológicas subsecuentes. 
Cuadro 4. Desviación estándar de la longitud de las ramas $(\mathrm{cm})$ y número de hojas de las ramas a los 19 dds (desarrollo vegetativo), a los 31 dds (madurez vegetativa) y a los 39 dds (floración) de verdolaga de la variedad 'Chapingo' sembrada en tres densidades de siembra (alta: 295; media: 250 y baja: 134 plantas $\mathrm{m}^{-1}$ lineal, respectivamente).

\begin{tabular}{|c|c|c|c|c|c|c|c|c|}
\hline \multirow{2}{*}{$\begin{array}{c}\text { Densidad } \\
\text { de } \\
\text { siembra }\end{array}$} & \multicolumn{2}{|r|}{$\mathrm{LPR}_{1}$} & \multicolumn{2}{|c|}{$\mathrm{NHP}_{1}$} & \multicolumn{2}{|r|}{$\mathrm{LPR}_{4}$} & \multicolumn{2}{|r|}{$\mathrm{NHP}_{4}$} \\
\hline & DS & $\begin{array}{c}\text { Levene } \\
\text { (valor F/Pr) }\end{array}$ & DS & $\begin{array}{c}\text { Levene } \\
\text { (valor F/Pr) }\end{array}$ & DS & $\begin{array}{c}\text { Levene } \\
\text { (valor F/Pr) }\end{array}$ & DS & $\begin{array}{c}\text { Levene } \\
\text { (valor F/Pr) }\end{array}$ \\
\hline \multicolumn{9}{|c|}{ Desarrollo vegetativo } \\
\hline Alta & 1.01 & $1.62 / 0.2072$ & 2.6 & $2.76 / 0.0721$ & 0.2 & $0.21 / 0.8091$ & 2.3 & $0.41 / 0.6638$ \\
\hline Media & 1.86 & & 3.6 & & 0.21 & & 2.51 & \\
\hline Baja & 2.09 & & 4.29 & & 0.25 & & 2.12 & \\
\hline \multicolumn{9}{|c|}{ Madurez vegetativa } \\
\hline Alta & 5.92 & $0.61 / 0.5485$ & 8.96 & $6.74 / 0.0023$ & 2.03 & $3.61 / 0.0334$ & 3.31 & $2.69 / 0.0767$ \\
\hline Media & 5.82 & & 14.15 & & 1.96 & & 5.6 & \\
\hline Baja & 4.28 & & 5.86 & & 3.66 & & 2.83 & \\
\hline \multicolumn{9}{|c|}{ Floración } \\
\hline Alta & 4.67 & $5.24 / 0.0082$ & 8.41 & $2.83 / 0.0675$ & 3.06 & $0.30 / 0.7406$ & 13.01 & $0.54 / 0.5857$ \\
\hline Media & 7.18 & & 9.1 & & 3.41 & & 10.76 & \\
\hline Baja & 10.01 & & 14.83 & & 3.57 & & 11.02 & \\
\hline
\end{tabular}

LPR $n=$ longitud promedio de las dos ramas del $n$ nudo; $\operatorname{NHP} n=$ número de hojas promedio de las dos ramas del $n$ nudo. Grupo de datos en negritas indican grupo de varianzas con diferencias estadísticamente significativas entre ellos $(p<0.05)$.

\section{Conclusiones}

Existen diferencias significativas entre densidades de siembra para altura total (AT), número de hojas chicas $(\mathrm{NHCH})$, longitud promedio de las dos ramas del nudo 1 y 4 (LPR 1 y $\mathrm{LPR}_{1}$, respectivamente) y número de hojas promedio de las dos ramas del nudo 1 ( $\left.\mathrm{NHP}_{1}\right)$. Altas densidades de siembra generan plantas con mayor altura, menor diámetro de tallo, menor número de hojas (grandes y chicas) y menor desarrollo de las ramas (longitud de ramas); sin embargo, el número de hojas desarrollado en las ramas depende del nivel del dosel donde éstas se localicen.

\section{Literatura citada}

Ayala, D. I. M.; Rey, B. L. y Durán, Q. C. 2004. Efecto de la densidad de siembra sobre el crecimiento, desarrollo y productividad de dos materiales de palma de aceite Elaeis guineensis Jacq. Rev. Palmas. 25:66-73. https://publicaciones.fedepalma.org/ index.php/palmas/article/view/1067.

Balcázar-Quiñones, A; White-Olascoaga, L.; Chávez-Mejía, C. y Zepeda-Gómez, C. 2020. Los quelites: riqueza de especies y conocimiento tradicional en la comunidad otomí de San Pedro Arriba, Temoaya, Estado de México. Polibotánica. 49:219-242. https://doi.org/10.18387/polibotanica.49.14. 
Bye, R. y Linares, E. 2002. Los quelites, plantas comestibles de México. Una reflexión sobre intercambio cultural. Biodiversitas. Comisión Nacional para el Conocimiento y Uso de la Biodiversidad (CONABIO). 31:11-14.

Díaz, L.; Viloria, Z. A. y Arteaga, R. L. 1999. Crecimiento vegetativo del pimentón en función de la densidad de plantas y edad del cultivo. Bioagro. 11(2):69-73.

Favaro, J. C. y Pilati R. A. 1997. Cultivo de tomate. In: cultivos bajo invernaderos. Centro de Publicaciones Universidad Nacional del Litoral y Editorial Hemisferio Sur. $2^{\text {da }}$ (Ed.). Buenos Aires, Argentina. 7-33 pp.

García, E. 1988. Los climas de México. Sistemas de Información Geográfica, SA. México, DF. 1-16 pp.

García, M. y Watson, C. E. 2003. Herencia de la resistencia al acame de raíces en maíz dulce (Zea mays L). Revista Científica UDO Agrícola. 3:24-33.

INEGI. 2015. Instituto Nacional de Estadística y Geografía. Encuesta Intercensal. Dirección General de Estadísticas Sociodemográficas. Encuesta Intercensal 2015. Instituto Nacional de Estadística y Geografía. México, DF. https://www.inegi.org.mx/programas/ intercensal/2015/.

Kakiuchi, J. and Kobata, R. 2004. Shading and thinning effects on seed and shoot dry matter increase in determinate soybean during the seed filling period. Agron. J. 96:398-405.

Mera, O. L. M.; Castro, D. L.; Bye, R. A. y Villanueva, V. C. 2010. Importancia de la verdolaga en México. Universidad Autónoma Chapingo. Chapingo, Estado de México. 28 p.

Montoya-García, C. O.; Volke-Haller, V. H.; Trinidad-Santos, A. and Villanueva-Verduzco, C. 2018. Change in the contents of fatty acids and antioxidant capacity of purslane in relation to fertilization. Scientia Horticulturae. 234:152-159.

Montoya-García, C. O.; Volke-Haller, V. H.; Trinidad-Santos, A.; Villanueva-Verduzco, C. y Sánchez-Escudero, J. 2017. Respuesta de la verdolaga (Portulaca oleracea L.) a la fertilización con NPK. Rev. Fitotec. Mex. 40(3):325-332.

Noda, Y. y Giraldo, M. 2008. Efecto de la densidad de siembra en el establecimiento de morera para su inclusión en sistemas ganaderos. Zootecnia Tropical. 26(3):339-341. http://ve.scielo.org/scielo.php?script=sci_arttext\&pid=S079872692008000300039\&lng=e s\&tlng=es.

Poulain, D. 1984. Influence of density on the growth and development of winter field bean (Vicia faba L.), 159-167 pp. In: Hebblethwaite, P. D.; Dawkins, T. C. K. Heath, M. C. and Lockwood. G. (Ed). Vicia faba: agronomy, phisiology and breeding. Martinus Nijhoff/ Dr. Junk, W. Publishers, Netherlands. 316 p.

Rodríguez, L. 2000. Densidad de población vegetal y producción de materia seca. Revista COMALFI. 27(1-2):31-38.

Santiago-Sáenz, Y. O.; Hernández-Fuentes, A. D.; López-Palestina, C. U.; Garrido-Cauich, J. H.; Alatorre-Cruz, J. M. y Monroy-Torres, R. 2019. Importancia nutricional y actividad biológica de los compuestos bioactivos de quelites consumidos en México. Revista Chilena de Nutrición. 46(5):593-605. https://dx.doi.org/10.4067/S0717-75182019000500593.

SAS Institute. 2002. SAS versión 9.1.3 help and documentation. Cary, NC, SAS Institute Inc $633 \mathrm{p}$.

SIAP. 2019. Servicio de Información Agroalimentaria y Pesquera. Producción Agrícola. Servicio de Información Agroalimentaria y Pesquera, SAGARPA. Ciudad de México. https://nube.siap.gob.mx/cierreagricola. 
Soil Survey Staff. 2010. Claves para la taxonomía de suelos. 11 ${ }^{\mathrm{a}}$ (Ed.). Ortiz-Solorio. C. A. and Gutiérrez-Castorena, M. C. (Trads.). USDA Natural Resources Conservation Service, Washington, DC. USA. 365 p.

Solís, A. G.; Mera, O. M.; Castro, L. D.; Pinzón, L. L.; Noguez, H. R.; Casanova, G. E. y Méndez, V.R. 2016. Conservación y aprovechamiento sostenible de especies vegetales tradicionales de México. Servicio Nacional de Inspección y Certificación de Semillas y Universidad Autónoma Chapingo. Chapingo, Estado de México. 108 p.

Villanueva, V. C. y Ramírez, A, M. 2003. Verdolaga hidropónica y tecnología de producción superintensiva de germinados en invernadero. In: Memorias del XIX Presentación de trabajos de investigación, producción y servicio. Departamento de Fitotecnia. Chapingo, Estado de México. 68-72 pp. 